\title{
Asymptotic expansions for Taylor coefficients of the composition of two functions
}

Article

Accepted Version

Hilberdink, T. (2009) Asymptotic expansions for Taylor coefficients of the composition of two functions. Asymptotic Analysis, 63 (3). pp. 125-142. ISSN 1875-8576 doi: https://doi.org/10.3233/ASY-2008-0926 Available at https://centaur.reading.ac.uk/23294/

It is advisable to refer to the publisher's version if you intend to cite from the work. See Guidance on citing.

To link to this article DOI: http://dx.doi.org/10.3233/ASY-2008-0926

Publisher: IOS Press

All outputs in CentAUR are protected by Intellectual Property Rights law, including copyright law. Copyright and IPR is retained by the creators or other copyright holders. Terms and conditions for use of this material are defined in the End User Agreement.

www.reading.ac.uk/centaur

\section{CentAUR}


Central Archive at the University of Reading

Reading's research outputs online 


\title{
Asymptotic expansions for Taylor coefficients of the composition of two functions ${ }^{1}$
}

\author{
Titus Hilberdink
}

Department of Mathematics, University of Reading, Whiteknights, PO Box 220, Reading RG6 6AX, England; t.w.hilberdink@reading.ac.uk

\begin{abstract}
We give an asymptotic expansion for the Taylor coefficients of $L(P(z))$ where $L(z)$ is analytic in the open unit disc whose Taylor coefficients vary 'smoothly' and $P(z)$ is a probability generating function. We show how this result applies to a variety of problems, amongst them obtaining the asymptotics of Bernoulli transforms and weighted renewal sequences.
\end{abstract}

Keywords: Asymptotic expansions, Taylor coefficients, weighted renewal sequences. AMS 2000 subject classification: 41A60, (30B10, 62E20).

\section{Introduction}

In this paper we investigate the asymptotic behaviour of the Taylor coefficients of the composition of two functions analytic in the unit disc; i.e. of

$$
F(z)=L(P(z)) .
$$

Equivalently, writing $f_{n}, l_{n}$ and $p_{n}$ for the Taylor coefficients of $F(z), L(z)$ and $P(z)$ respectively, this is

$$
f_{n}=\sum_{m=0}^{\infty} l_{m} p_{n}^{* m}
$$

(Here $p_{n}^{* m}$ is the $m^{\text {th }}$-convolution of $p_{n}$.) Throughout this paper, $P(z)$ is a probability generating function; i.e. $p_{n} \geq 0$ and $\sum p_{n}=1$. In probability theory, sequences of the form $(1.2)$ are called weighted renewal sequences (with 'weights' $l_{n}$ ). They have been studied by many authors (see for example [4], [11], [7] and the references in [2]).

In [8], it was shown that if $P(z)$ is not of the form $k\left(z^{r}\right)$ (for some holomorphic function $k(\cdot)$ and integer $r \geq 2), p_{n}=O\left(e^{-\lambda \sqrt{n}}\right)$ for some $\lambda>0$, and

$$
l_{n+[\lambda \sqrt{n}]} \sim l_{n}
$$

as $n \rightarrow \infty$ for all $\lambda$, then

$$
f_{n} \sim \sigma l_{[\sigma n]}
$$

as $n \rightarrow \infty$, where $\sigma=1 / \sum_{n=1}^{\infty} n p_{n}$.

It is natural to consider whether one can obtain more precise information about the asymptotic behaviour of $f_{n}$ or, if possible, find an asymptotic expansion for $f_{n}$ in terms of the $l_{[\sigma n]}$. This is often needed in problems related to probability theory, combinatorics and information theory. See for example [12] for a wide range of problems on asymptotic

\footnotetext{
${ }^{1}$ Asymptotic Analysis 63 (2009) 125-142.
} 
enumeration in combinatorics and [2] for examples in probability theory. Detailed asymptotic estimates are also needed in entropy computations and other problems in information theory (see for example [9],[10]).

In an interesting recent development, Baltrūnas and Šiaulys ([1]) obtained a bound on the difference $f_{n}-\sigma l_{[\sigma n]}$ for a particular class of $l_{n}$ which were shown to satisfy (1.3). They considered the case where the $l_{n}$ are of the form $e^{-H_{n}}$ with $H_{n}-H_{n-1}$ decreasing and $n\left(H_{n}-H_{n-1}\right) \leq\left(\frac{1}{2}-\delta\right) H_{n}$ for some $\delta>0$ (for large $n$ ). They found that

$$
f_{n}=\sigma l_{[\sigma n]}\left(1+O\left(\frac{H_{n} \log n}{\sqrt{n}}\right)\right)
$$

Our goal in this paper is to give an asymptotic expansion of the coefficients $f_{n}$. In order to do this, we need to have more precise information of the behaviour of $l_{n}$ for large $n$. By interpolation, we may extend $l_{n}$ to an infinitely differentiable function $l(x)$ for real $x \geq 0$. Then, on condition that the behaviour of the higher derivatives of $l(x)$ are not too wild, we obtain the following asymptotic expansion of $f_{n}$ :

$$
f_{n} \approx q_{0}(n) l(\sigma n)+q_{1}(n) l^{\prime}(\sigma n)+\ldots+q_{k}(n) l^{(k)}(\sigma n)+\ldots,
$$

where the $q_{k}(n)$ are polynomials of degree $[k / 2]$ which can be explicitly calculated from $P(z)$; eg. $q_{0}(n)=\sigma$. The methods that we use here are similar to those in [8] but give a more detailed analysis of the expansion of $f_{n}$ in terms of the Taylor expansion of $l(x)$.

In our final section, we apply our results to a variety of problems. We consider the asymptotic behaviour of Bernoulli transforms as introduced in [5]. The asymptotic behaviour is easily derived and in greater generality. Furthermore, our methods do not require the extra analyticity conditions necessary for singularity analysis. Similarly, we treat the negative binomial sums discussed in [9]. Finally, we see how our results impact on the results of [1].

\section{Conditions and Statement of main result}

Following [2], we call a sequence $\left\{p_{n}\right\}$ of probabilities aperiodic if

$$
\operatorname{supp}\left(p_{n}\right):=\left\{n \in \mathbb{N}: p_{n}>0\right\} \not \subset q \mathbb{N} \quad \text { for any integer } q>1 .
$$

(It is periodic with period $q>1$ if $\operatorname{supp}\left(p_{n}\right) \subset q \mathbb{N}$ with $q$ maximal.) We say $\left\{p_{n}\right\}$ is non-lattice if

$$
\operatorname{supp}\left(p_{n}\right) \not \subset q \mathbb{N}_{0}+p \quad \text { for any } q>1 \text { and } p \in\{1, \ldots, q\} .
$$

Note that $(\mathrm{P} 1)$ is equivalent to saying that $P(z)$ is not of the form $k\left(z^{q}\right)$ for any $k(\cdot)$ holomorphic. It also implies that $P(z)=1$ at $z=1$ only (on the unit circle). The stronger condition (P2) is equivalent to saying that $P(z)$ is not of the form $z^{p} k\left(z^{q}\right)$ for any $k(\cdot)$ holomorphic. It implies that $|P(z)|=1$ at $z=1$ only (on the unit circle).

As in [8], we assume that

$$
p_{n}=O\left(e^{-\lambda \sqrt{n}}\right) \quad \text { for some } \lambda>0 .
$$

In particular, this implies the convergence of $\sum n^{k} p_{n}$ for every $k$.

Regarding the function $L(z)=\sum_{n=0}^{\infty} l_{n} z^{n}$, we shall assume that $l_{n}$ is eventually positive and satisfies

$$
l_{n+[\lambda \sqrt{n}]} \sim l_{n}
$$


as $n \rightarrow \infty$, for all real $\lambda$. We note that (L1) is equivalent to $l_{n}=s\left(e^{\sqrt{n}}\right)$ for some slowlyvarying function $s(\cdot)$ (see [8]), hence $l_{n}=e^{o(\sqrt{n})}$ and $L(z)$ is analytic for $|z|<1$.

Let $f_{n}$ be the coefficient of $z^{n}$ in the power series expansion of the composite function $F(z)=L(P(z))$. By expanding $L(z)$, it is seen that $f_{n}$ is given by (1.2). The implication

$$
(\mathrm{P} 1),(\mathrm{P} 3) \text { and }(\mathrm{L} 1) \Longrightarrow f_{n} \sim \sigma l_{[\sigma n]}
$$

was proven in [8].

In order to obtain an asymptotic expansion for $f_{n}$ it is necessary to make extra assumptions about the sequence $l_{n}$. We may extend $l_{n}$ to an infinitely differentiable function $l(x)$ on $\mathbb{R}_{\geq 0}$ by interpolation. We shall assume that for a given $r \geq 1$,

$$
l^{(k)}(x+t)=O\left(\left|l^{(k)}(x)\right|\right) \quad \text { for } t=O(\sqrt{x} \log x),
$$

for $k=1, \ldots, r$, the implied constant being independent of $t$. (In fact, we can weaken this condition to $t=O(\sqrt{x \log x})$ if $P(z)$ is analytic beyond the unit disc). Thus, by Taylor's theorem, (L2) implies

$$
l(x+t)=l(x)+t l^{\prime}(x)+\frac{t^{2} l^{\prime \prime}(x)}{2 !}+\ldots+\frac{t^{r-1} l^{(r-1)}(x)}{(r-1) !}+O\left(\left|t^{r} l^{(r)}(x)\right|\right)
$$

for $t=O(\sqrt{x} \log x)$.

Our main result is

\section{Theorem 1}

Let $P(z)=\sum_{n=0}^{\infty} p_{n} z^{n}$ where $\left\{p_{n}\right\}$ satisfies (P1) and (P3). Let $L(z)=\sum_{n=0}^{\infty} l_{n} z^{n}$ where $\left\{l_{n}\right\}$ satisfies (L1) and (L2). Then the Taylor coefficients $f_{n}$ of $F(z)=L(P(z))$ have the asymptotic development

$$
f_{n}=\sum_{k=0}^{r-1} q_{k}(n) l^{(k)}(\sigma n)+O\left(n^{r / 2}\left|l^{(r)}(\sigma n)\right|\right)+O\left(\frac{l(\sigma n)}{n^{A}}\right) \quad \quad(\text { for all } A)
$$

where $\sigma=1 / \sum_{n=1}^{\infty} n p_{n}$ and the $q_{k}(n)$ are polynomials of degree $[k / 2]$.

It is elementary to show that if (L1) and (L2) are satisfied, then $l^{(k)}(x)=o\left(l(x) x^{-k / 2}\right)$ for $k=1, \ldots, r$, so this is genuinely an asymptotic expansion.

As will be shown in section 4 , the polynomials $q_{k}(n)$ can be calculated explicitly from $P(z)$ via the formula ${ }^{2}$

$$
q_{k}(n)=\left[\frac{1}{t}\right] \frac{(-1)^{k} P(1+t)^{\sigma n}}{(\log P(1+t))^{k+1}(1+t)^{n+1}} .
$$

\section{Proofs}

The idea of the proof of Theorem 1 is to show that the main contribution towards $f_{n}$ in (1.2) comes from the range $m=\sigma n+O(\sqrt{n} \log n)$ in the sense that

$$
f_{n}=\sum_{m=\sigma n+O(\sqrt{n} \log n)} l_{m} p_{n}^{* m}+O\left(\frac{l_{[\sigma n]}}{n^{A}}\right) \quad \text { for every } A .
$$

\footnotetext{
${ }^{2}$ Here, and elsewhere, $\left[t^{n}\right] H(t)$ (with $n \in \mathbb{Z}$ ) means the coefficient of $t^{n}$ in the expansion (asymptotic or otherwise) of $H(t)$ for $t$ small.
} 
Condition (L2) then allows us to 'expand' $l_{m}$ as

$$
l_{m}=l(\sigma n+m-\sigma n) \approx \sum_{k=0}^{r-1} \frac{l^{(k)}(\sigma n)}{k !}(m-\sigma n)^{k}
$$

in order to obtain

$$
f_{n} \approx \sum_{k=0}^{r-1} \frac{l^{(k)}(\sigma n)}{k !} \sum_{m=\sigma n+O(\sqrt{n} \log n)}(m-\sigma n)^{k} p_{n}^{* m} .
$$

We therefore need an estimate for the inner sum on the right. These follow estimates for sums of the form $\sum_{m=0}^{\infty} m^{k} p_{n}^{* m}$. Thus we first prove the following:

\section{Proposition 2}

Let $P(z)=\sum_{n=0}^{\infty} p_{n} z^{n}$ with $p_{n} \geq 0$, aperiodic, $p_{n}=O\left(n^{-A}\right)$ for all $A$ and $P(1)=1$. Then, for $k \in \mathbb{N}_{0}$,

$$
\sum_{m=0}^{\infty} m^{k} p_{n}^{* m}=Q_{k}(n)+O\left(\frac{1}{n^{A}}\right) \quad(\text { for all } A)
$$

where $Q_{k}(n)$ is a polynomial of degree $k$. (Here and elsewhere $0^{0}=1$ ).

Proof. First let us suppose that $P(z)$ is analytic beyond the unit disc. Note that $\sum_{m=0}^{\infty} m^{k} p_{n}^{* m}$ is the coefficient of $z^{n}$ in the expansion in $R_{k}(P(z))$ where

$$
R_{k}(z)=\sum_{r=0}^{\infty} r^{k} z^{r}
$$

It is elementary to show that we may write $R_{k}(z)=\frac{r_{k}(z)}{(1-z)^{k+1}}$ where $r_{k}(\cdot)$ is a polynomial of degree $k$. Thus

$$
\begin{aligned}
\sum_{m=0}^{\infty} m^{k} p_{n}^{* m} & =\frac{1}{2 \pi i} \int_{\gamma} \frac{r_{k}(P(z))}{z^{n+1}(1-P(z))^{k+1}} d z \\
& =-\operatorname{Res}_{z=1}\left\{\frac{r_{k}(P(z))}{z^{n+1}(1-P(z))^{k+1}}\right\}+O\left(a^{-n}\right)
\end{aligned}
$$

for some $a>1$, by taking $\gamma$ past the pole at $z=1$. Note that here we use the fact that $P(z)=1$ at $z=1$ only for $|z| \leq 1$ (and hence for $|z|<a$, for some $a>1$ ). Consider the residue and let $z=1+w$.

$$
\begin{aligned}
\frac{r_{k}(P(1+w))}{(1+w)^{n+1}(1-P(1+w))^{k+1}} & =(-1)^{k+1} \frac{r_{k}\left(1+P^{\prime}(1) w+\ldots\right)(1+w)^{-(n+1)}}{\left(P^{\prime}(1) w+\frac{1}{2} P^{\prime \prime}(1) w^{2}+\ldots\right)^{k+1}} \\
& =\frac{(-1)^{k+1}(A+B w+\ldots)}{w^{k+1} P^{\prime}(1)^{k+1}(1+C w+\ldots)} \sum_{r=0}^{\infty}(-1)^{r}\left(\begin{array}{c}
n+r \\
r
\end{array}\right) w^{r}
\end{aligned}
$$

for some $A, B, C \ldots$ By multiplying out the series, it is easily seen that the coefficient of $1 / w$ in this expansion is some polynomial in $n$ of degree $k$. Thus

$$
\sum_{m=0}^{\infty} m^{k} p_{n}^{* m}=Q_{k}(n)+O\left(a^{-n}\right)
$$


where $Q_{k}$ is a polynomial of degree $k$. Note that the error term is much smaller than that stated in the theorem; this is due to the extra analyticity assumption on $P(\cdot)$. had

If $P(z)$ is not analytic beyond the unit disc we have to be more subtle. From above we

$$
\sum_{m=0}^{\infty} m^{k} p_{n}^{* m}=\left[z^{n}\right] \frac{r_{k}(P(z))}{(1-P(z))^{k+1}}
$$

where $r_{k}(\cdot)$ is some polynomial of degree $k$. Under the assumptions, $\sum_{n=0}^{\infty} p_{n} n^{r}$ converges for all $r \geq 0$, and so we have ${ }^{3}$

$$
P(z)=1-P^{\prime}(1)(1-z)+\frac{P^{\prime \prime}(1)}{2 !}(1-z)-\ldots+\frac{(-1)^{N} P^{(N)}(1)}{N !}(1-z)^{N}+O\left((1-z)^{N+1}\right)
$$

as $z \rightarrow 1(|z| \leq 1)$ for any $N$. Since $r_{k}(\cdot)$ is a polynomial we have the asymptotic expansion

$$
\frac{r_{k}(P(z))}{(1-P(z))^{k+1}}=\frac{A_{0}}{(1-z)^{k+1}}+\ldots+\frac{A_{k}}{1-z}+\ldots+A_{N}(1-z)^{N-k-1}+O\left((1-z)^{N-k}\right)
$$

as $z \rightarrow 1$, for some $A_{i}$ (depending on $i$ and $k$ only). Let

$$
g(z)=\frac{r_{k}(P(z))}{(1-P(z))^{k+1}}-\sum_{r=0}^{k} \frac{A_{r}}{(1-z)^{k+1-r}}
$$

for $|z| \leq 1, z \neq 1$. (This is well-defined since $P(z)=1$ at $z=1$ only, in the closed unit disc.) Now, the function $P\left(e^{i \theta}\right)$ is infinitely differentiable (for real $\theta$ ). It follows that $r_{k}\left(e^{i \theta}\right) /\left(1-P\left(e^{i \theta}\right)\right)^{k+1}$, and hence $g\left(e^{i \theta}\right)$, are infinitely differentiable on $(0,2 \pi)$. But from the above we have

$$
g\left(e^{i \theta}\right)=\beta_{0}+\beta_{1} \theta+\ldots+\beta_{N} \theta^{N}+O\left(\theta^{N+1}\right) \quad \text { as } \theta \rightarrow 0
$$

for some $\beta_{i}$ and any $N$. This asymptotic expansion may be differentiated since, by the same arguments,

$$
g^{\prime}\left(e^{i \theta}\right)=\gamma_{0} \theta^{m}+\gamma_{1} \theta^{m+1}+\ldots+\gamma_{N} \theta^{N+m}+O\left(\theta^{N+m+1}\right) \quad \text { as } \theta \rightarrow 0
$$

for some $m \in \mathbb{Z}$ and $\gamma_{i}$, and integrating would have to yield (3.1). Repeating this argument, (3.1) may be differentiated any number of times and by defining $\left.\frac{d^{k}}{d \theta^{k}} g\left(e^{i \theta}\right)\right|_{\theta=0}=k ! \beta_{k}$, it follows that $g\left(e^{i \theta}\right)$ is extendable to an infinitely differentiable function on $\mathbb{R}$. Integrating by parts $N$ times gives

$$
\begin{aligned}
{\left[z^{n}\right] g(z) } & =\frac{1}{2 \pi} \int_{0}^{2 \pi} g\left(e^{i \theta}\right) e^{-n i \theta} d \theta=\frac{1}{2 \pi n} \int_{0}^{2 \pi} g^{\prime}\left(e^{i \theta}\right) e^{-(n-1) i \theta} d \theta= \\
\ldots= & \frac{1}{2 \pi n(n-1) \ldots(n-N+1)} \int_{0}^{2 \pi} g^{(N)}\left(e^{i \theta}\right) e^{-(n-N) i \theta} d \theta=O\left(\frac{1}{n^{N}}\right)
\end{aligned}
$$

as $n \rightarrow \infty$, for every $N$. Hence

$$
\left[z^{n}\right] \frac{r_{k}(P(z))}{(1-P(z))^{k+1}}=\left[z^{n}\right] \sum_{r=0}^{k} \frac{A_{r}}{(1-z)^{k+1-r}}+g(z)=Q_{k}(n)+O\left(\frac{1}{n^{N}}\right)
$$

\footnotetext{
${ }^{3}$ For convenience we shall write $P^{(k)}(1)=\sum_{n=k}^{\infty} n(n-1) \ldots(n-k+1) p_{n}$. Thus $\sigma=1 / P^{\prime}(1)$.
} 
where $Q_{k}(n)$ is a polynomial of degree $k$. Note that the polynomials $Q_{k}(n)$ necessarily coincide with the earlier ones since their coefficients are identical combinations of $P^{(r)}(1)$.

Now we need a sharper version of Lemma 2.4 of [8]. It was observed in [8] that $P\left(e^{z}\right)=$ $\exp \left\{\frac{z}{\sigma}+\tau z^{2}+O\left(z^{3}\right)\right\}$ as $z \rightarrow 0$, where $\tau=\frac{1}{2}\left(P^{\prime}(1)+P^{\prime \prime}(1)-P^{\prime}(1)^{2}\right)>0$. From this we obtain the inequality

$$
\left|P\left(e^{x+i y}\right)\right| \leq \exp \left\{\frac{x}{\sigma}+a x^{2}-b y^{2}\right\}
$$

for $x, y$ real and sufficiently small, which holds for some $a, b>0$.

For the following Lemma we shall, for simplicity of presentation, assume the stronger condition (P2) rather than (P1) throughout. We mention the necessary adjustments to be made if only (P1) is assumed, afterwards.

Let $\Delta=m-\sigma n$.

\section{Lemma 3}

Suppose that $P(z)$ is analytic for $|z|<1+\delta$ for some $\delta>0$ and (P2) holds. Then

(i) For $0 \leq m \leq(\sigma-\eta) n$ and $m \geq(\sigma+\eta) n$ (any $\eta>0)$, we have

$$
p_{n}^{* m} \leq e^{-\eta_{1} n}, e^{-\eta_{2} m} \quad \text { respectively for some } \eta_{1}, \eta_{2}>0 .
$$

(ii) For $(\sigma-\eta) n \leq m \leq(\sigma+\eta) n$ (i.e. $|\Delta| \leq \eta n)$, we have

$$
p_{n}^{* m}=O\left(\frac{1}{\sqrt{n}} e^{-\frac{\lambda \Delta^{2}}{n}}\right) \quad \text { for some } \lambda>0 .
$$

Proof. (i). Consider first $0 \leq m \leq(\sigma-\eta) n$. We have

$$
0 \leq p_{n}^{* m} \leq \frac{P(r)^{m}}{r^{n}}
$$

for any $0<r<1+\delta$ since $|P(z)| \leq P(|z|)$. Take $r=e^{\varepsilon}$ where $\varepsilon>0$ is chosen such that

$$
P\left(e^{\varepsilon}\right) \leq \exp \left\{\frac{\varepsilon}{\sigma}\left(1+\frac{\eta}{2(\sigma-\eta)}\right)\right\} .
$$

This is possible because $P\left(e^{t}\right)=\exp \left(\frac{1}{\sigma} t+O\left(t^{2}\right)\right)$ as $t \rightarrow 0$. Then

$$
p_{n}^{* m} \leq \exp \left\{\frac{\varepsilon m}{\sigma}\left(1+\frac{\eta}{2(\sigma-\eta)}\right)-\varepsilon n\right\} \leq \exp \left\{\frac{(\sigma-\eta) \varepsilon n}{\sigma}\left(1+\frac{\eta}{2(\sigma-\eta)}\right)-\varepsilon n\right\}=e^{-\eta_{1} n}
$$

with $\eta_{1}=\frac{\eta \varepsilon}{2 \sigma}>0$ as required. For $m \geq(\sigma+\eta) n$, take $r=e^{-\varepsilon}$ where $\varepsilon>0$ is chosen such that

Then

$$
P\left(e^{-\varepsilon}\right) \leq \exp \left\{-\frac{\varepsilon}{\sigma}\left(1-\frac{\eta}{2(\sigma+\eta)}\right)\right\} .
$$

$$
p_{n}^{* m} \leq \exp \left\{\varepsilon n-\frac{\varepsilon m}{\sigma}\left(1-\frac{\eta}{2(\sigma+\eta)}\right)\right\} \leq \exp \left\{\frac{\varepsilon m}{\sigma+\eta}-\frac{\varepsilon m}{\sigma}\left(1-\frac{\eta}{2(\sigma+\eta)}\right)\right\}=e^{-\eta_{2} m},
$$


with $\eta_{2}=\frac{\eta \varepsilon}{2 \sigma(\sigma+\eta)}>0$.

(ii). For the range $|\Delta| \leq \eta n$, we use Cauchy's formula

$$
p_{n}^{* m}=\frac{1}{2 \pi i} \int_{\gamma} \frac{P(z)^{m}}{z^{n+1}} d z=\frac{1}{2 \pi r^{n}} \int_{0}^{2 \pi} e^{-n i \theta} P\left(r e^{i \theta}\right)^{m} d \theta .
$$

Choose $r=e^{-\lambda \Delta / n}$ and $\delta_{n}=\sqrt{\frac{B \log n}{n}}$ (with $\lambda, B>0$ small and large respectively to be determined). We split the integral into two parts:

$$
p_{n}^{* m}=\frac{1}{2 \pi r^{n}} \int_{-\delta_{n}}^{\delta_{n}} e^{-n i \theta} P\left(r e^{i \theta}\right)^{m} d \theta+\frac{1}{2 \pi r^{n}} \int_{\delta_{n}}^{2 \pi-\delta_{n}} e^{-n i \theta} P\left(r e^{i \theta}\right)^{m} d \theta .
$$

Call these integrals $I_{1}$ and $I_{2}$ respectively. We have $\left|I_{2}\right| \leq r^{-n}\left|P\left(r e^{ \pm i \delta_{n}}\right)\right|^{m}$ for all $n$ sufficiently large, since $\left|P\left(r e^{i \theta}\right)\right|$ increases as $\theta$ gets closer to 0 and $2 \pi$, where it is maximal. Hence, using (3.2) and substituting $r=e^{-\lambda \Delta / n}$ and $\delta_{n}=\sqrt{B \log n} / \sqrt{n}$, we get

$$
\begin{aligned}
\left|I_{2}\right| & \leq \exp \left\{\lambda \Delta-\frac{\lambda \Delta m}{\sigma n}+\frac{a \lambda^{2} \Delta^{2} m}{n^{2}}-b m \delta_{n}^{2}\right\} \\
& \leq \exp \left\{\lambda \Delta-\frac{\lambda \Delta(\sigma n+\Delta)}{\sigma n}+\frac{a \lambda^{2} \Delta^{2}(\sigma+\eta) n}{n^{2}}-b(\sigma-\eta) n \delta_{n}^{2}\right\} \\
& =\exp \left\{-\frac{\lambda \Delta^{2}}{\sigma n}+\frac{a \lambda^{2}(\sigma+\eta) \Delta^{2}}{n}-b(\sigma-\eta) B \log n\right\}=O\left(\frac{1}{\sqrt{n}} e^{-\lambda_{1} \Delta^{2} / n}\right)
\end{aligned}
$$

where $\lambda_{1}=\frac{\lambda}{\sigma}(1-a \sigma(\sigma+\eta) \lambda)>0$ by choosing $\lambda$ sufficiently small, and by taking $B$ sufficiently large. For $\left|I_{1}\right|$, we have the bound

$$
\begin{aligned}
\left|I_{1}\right| & \leq \frac{1}{2 \pi r^{n}} \int_{-\delta_{n}}^{\delta_{n}}\left|P\left(r e^{i \theta}\right)\right|^{m} d \theta \\
& \leq \frac{e^{\lambda \Delta}}{2 \pi} \int_{-\delta_{n}}^{\delta_{n}} \exp \left\{-\frac{\lambda \Delta m}{\sigma n}+\frac{a \lambda^{2} \Delta^{2} m}{n^{2}}-b \theta^{2} m\right\} d \theta \\
& \leq \frac{1}{2 \pi} \exp \left\{-\frac{\lambda \Delta^{2}}{\sigma n}+\frac{a(\sigma+\eta) \lambda^{2} \Delta^{2}}{n}\right\} \int_{-\infty}^{\infty} e^{-b(\sigma-\eta) n \theta^{2}} d \theta \\
& =O\left(\frac{1}{\sqrt{n}} e^{-\lambda_{1} \Delta^{2} / n}\right)
\end{aligned}
$$

as desired.

Remark. If we only assume (P1) then the result still holds, but the proof of (ii) must be adjusted. Specifically, we need to consider the case where $P(z)=z^{p} k\left(z^{q}\right)$ for some $p, q$ coprime with $q>1$. In this case $P(\eta z)=\eta^{p} P(z)$ for every $q^{\text {th }}$-root of unity $\eta$, and $|P(z)|=1$ precisely at the $q^{\text {th }}$-roots of unity. Then (3.2) becomes $\mid P\left(\eta e^{x+i y} \mid\right) \leq \exp \left\{\frac{x}{\sigma}+a x^{2}-b y^{2}\right\}$ and for (ii), we need to split the integral at each $q^{\text {th }}$-root of unity. 
Recall Corollary 2.3 of [8]: if $l_{n}=s\left(e^{\sqrt{n}}\right)$ with $s$ slowly-varying, then for $n$ and $n+k \rightarrow \infty$, we have

$$
l_{n+k}=l_{n} \exp \left\{o\left(\frac{|k|}{\sqrt{n}}\right)+o(1)\right\} .
$$

Proof of Theorem 1. We shall first consider the case where $P(z)$ is analytic beyond the unit disc and afterwards adjust the proof to cover the more difficult case where we only assume $p_{n}=O\left(e^{-\lambda \sqrt{n}}\right)$ for some $\lambda>0$. In this case we need only assume (L2) for $t=O(\sqrt{x \log x})$.

Choose $A$ (large) and let $S=\left\{m: \frac{\sigma}{2} n \leq m \leq 2 \sigma n\right.$ and $\left.|m-\sigma n| \geq A \sqrt{n \log n}\right\}$. Then from the bounds in Lemma 3 we find that

$$
\begin{aligned}
\sum_{|m-\sigma n| \geq A \sqrt{n \log n}} l_{m} p_{n}^{* m} & =\sum_{0 \leq m \leq \frac{\sigma n}{2}, m \geq 2 \sigma n} l_{m} p_{n}^{* m}+\sum_{m \in S} l_{m} p_{n}^{* m} \\
& \leq e^{-\eta_{1} n} \sum_{0 \leq m \leq \frac{\sigma n}{2}} l_{m}+\sum_{m \geq 2 \sigma n} l_{m} e^{-\eta_{2} m}+O\left(\frac{1}{\sqrt{n}} \sum_{m \in S} l_{m} e^{-\frac{\lambda \Delta^{2}}{n}}\right) \\
& \leq e^{-\eta^{\prime} n}+O\left(l(\sigma n) \sum_{k \geq A \sqrt{n \log n}} e^{-\frac{\lambda k^{2}}{n}+o\left(\frac{k}{\sqrt{n}}\right)}\right)
\end{aligned}
$$

by putting $k=m-[\sigma n]$, using the fact that $l_{n}=e^{o(\sqrt{n})}$, and (3.3). The last sum is bounded for all large $n$ by

$$
\int_{A \sqrt{n \log n}}^{\infty} e^{-\frac{\lambda t^{2}}{2 n}} d t=\sqrt{\frac{2 n}{\lambda}} \int_{A_{1} \sqrt{\log n}}^{\infty} e^{-y^{2}} d y=o\left(n^{-A_{1}^{2}+1 / 2}\right),
$$

where $A_{1}=A \sqrt{\lambda / 2}$. Writing $A_{2}=A_{1}^{2}-1 / 2$, we have

$$
f_{n}=\sum_{|\Delta| \leq A \sqrt{n \log n}} l_{m} p_{n}^{* m}+O\left(\frac{l(\sigma n)}{n^{A_{2}}}\right) .
$$

Note that since $A$ can be chosen arbitrarily large, both $A_{1}$ and $A_{2}$ can be arbitrarily large. Now use the Taylor approximation

$$
\left.l_{m}=l(\sigma n+\Delta)=\sum_{k=0}^{r-1} \frac{l^{(k)}(\sigma n) \Delta^{k}}{k !}+O\left|l^{(r)}(\sigma n) \Delta^{r}\right|\right),
$$

and substitute into (3.4) to obtain

$$
f_{n}=\sum_{k=0}^{r-1} \frac{l^{(k)}(\sigma n)}{k !} \sum_{|\Delta| \leq A \sqrt{n \log n}} \Delta^{k} p_{n}^{* m}+O\left(\left|l^{(r)}(\sigma n)\right| \sum_{|\Delta| \leq A \sqrt{n \log n}}|\Delta|^{r} p_{n}^{* m}\right)+O\left(\frac{l(\sigma n)}{n^{A_{2}}}\right) .
$$

It remains to find the asymptotic development of the sums $\sum \Delta^{k} p_{n}^{* m}$. But this follows from Proposition 2. For we have

$$
\begin{aligned}
\sum_{|\Delta| \leq A \sqrt{n \log n}} \Delta^{k} p_{n}^{* m} & =\sum_{|\Delta| \leq A \sqrt{n \log n}}(m-\sigma n)^{k} p_{n}^{* m}=\sum_{l=0}^{k}\left(\begin{array}{l}
k \\
l
\end{array}\right)(-\sigma n)^{k-l} \sum_{|\Delta| \leq A \sqrt{n \log n}} m^{l} p_{n}^{* m} \\
& =\sum_{l=0}^{k}\left(\begin{array}{l}
k \\
l
\end{array}\right)(-\sigma n)^{k-l}\left(\sum_{m=0}^{\infty} m^{l} p_{n}^{* m}\right)+O\left(\frac{1}{n^{A_{2}-k}}\right)
\end{aligned}
$$


by the arguments preceding (3.4). Proposition 2 then implies

$$
\sum_{|\Delta| \leq A \sqrt{n \log n}} \Delta^{k} p_{n}^{* m}=\sum_{l=0}^{k}\left(\begin{array}{l}
k \\
l
\end{array}\right)(-\sigma n)^{k-l} Q_{l}(n)+O\left(\frac{1}{n^{A_{2}-k}}\right) .
$$

Let

$$
q_{k}(n)=\frac{1}{k !} \sum_{l=0}^{k}\left(\begin{array}{l}
k \\
l
\end{array}\right)(-\sigma n)^{k-l} Q_{l}(n) .
$$

Thus $q_{k}(\cdot)$ is a polynomial of degree at most $k$. But by Lemma 3(ii),

$$
\begin{aligned}
\left|\sum_{|\Delta| \leq A \sqrt{n \log n}} \Delta^{k} p_{n}^{* m}\right| & \leq \frac{c}{\sqrt{n}} \sum_{|\Delta| \leq A \sqrt{n \log n}}|\Delta|^{k} e^{-\frac{\lambda \Delta^{2}}{n}}=O\left(\frac{1}{\sqrt{n}} \int_{0}^{\infty} t^{k} e^{-\frac{\lambda t^{2}}{n}} d t\right) \\
& =O\left(n^{k / 2} \int_{0}^{\infty} y^{(k-1) / 2} e^{-y} d y\right)=O\left(n^{k / 2}\right) .
\end{aligned}
$$

Hence the degree of $q_{k}(n)$ is at most $[k / 2]$. (Note that $p_{n}^{* m} \approx \frac{1}{2 \sqrt{\pi \sigma \tau n}} e^{-\Delta^{2} / 4 \sigma^{3} \tau n}$ ([8], Lemma $2.5)$ which is even in $\Delta$, so the different behaviour depending on the parity of $k$ is expected.) From (3.5) we therefore have

$$
f_{n}=\sum_{k=0}^{r-1} l^{(k)}(\sigma n) q_{k}(n)+O\left(n^{r / 2}\left|l^{(r)}(\sigma n)\right|\right)+O\left(\frac{l(\sigma n)}{n^{A}}\right) \quad(\text { for all } A),
$$

which proves the theorem for the case where $P(z)$ is analytic beyond the unit disc.

Now we complete the proof of Theorem 1 under the weaker condition $p_{n}=O\left(e^{-\lambda \sqrt{n}}\right)$ for some $\lambda>0$. The analysis becomes more difficult, since we cannot venture outside the unit disc to obtain bounds for $p_{n}^{* m}$.

The arguments used in Lemma 3 only hold for $m \geq \sigma n$ and fail for $m<\sigma n$. However, from [8] (p. 200), it was shown that

$$
\sum_{m<\sigma n-A \sqrt{n} \log n} l_{m} p_{n}^{* m}=O\left(\frac{l(\sigma n)}{n^{\frac{\mu A}{2 \sigma}-\frac{1}{2}}}\right) .
$$

Combining this with the bounds for $m \geq \sigma n$ gives

$$
f_{n}=\sum_{|\Delta| \leq A \sqrt{n} \log n} l_{m} p_{n}^{* m}+O\left(\frac{l(\sigma n)}{n^{A^{\prime}}}\right)
$$

where $A^{\prime}=\min \left\{\frac{\mu A}{2 \sigma}-\frac{1}{2}, A_{2}\right\}$ - this can also be made arbitrarily large. Note that the range for $\Delta$ is now $O(\sqrt{n} \log n)$ (compare (3.7) to (3.4)) and hence we require (L2) for $t=O(\sqrt{x} \log x)$. Under this mildly stronger (L2), we therefore obtain (3.5) again but with $A^{\prime}$ in place of $A_{2}$ and $\sqrt{n} \log n$ in place of $\sqrt{n \log n}$. The asymptotic behaviour of the sums $\sum \Delta^{k} p_{n}^{* m}$ follows that of $\sum m^{k} p_{n}^{* m}$ as before, thus:

$$
\sum_{|\Delta| \leq A \sqrt{n} \log n} \Delta^{k} p_{n}^{* m}=k ! q_{k}(n)+O\left(\frac{1}{n^{A^{\prime}-k}}\right) .
$$


Combining with (3.5) proves Theorem 1.

\section{Remarks 5}

(a) Note that for the purposes of obtaining the main terms in the asymptotic expansion, it is not necessary to find the $q_{k}(n)$ completely. For example, suppose $l^{(k)}(x)=$ $O\left(l(x) x^{-k}\right)$ as $x \rightarrow \infty$ for each $k$, which occurs typically when $l_{n}$ is regularly-varying. Then, to find an approximation with relative error $O\left(n^{-3}\right)$, we only need $q_{0}(n), q_{1}(n), q_{2}(n)$ and the leading terms from $q_{3}(n)$ and $q_{4}(n)$.

(b) Condition (L2) for $t=O(\sqrt{x} \log x)$ does not restrict the size of $l_{n}$ by much. Since $l_{n}=s\left(e^{\sqrt{n}}\right)$, we already have $l_{n}=e^{o(\sqrt{n})}$. But (L2) holds for sequences like $l_{n}=e^{n^{\alpha}}$ with $0<\alpha<1 / 2$. The slightly smaller range $t=O(\sqrt{x \log x})$ seems necessary since we need the full range $|\Delta|=O(\sqrt{n \log n})$ for an asymptotic expansion. This is because $p_{n}^{* m}$ can be as large as $\frac{c}{\sqrt{n}} e^{-\lambda|\Delta|^{2} / n}=c n^{-\lambda a^{2}-1 / 2}$ when $\Delta=a \sqrt{n \log n}$ (some $\lambda, c>0$ ).

(c) Discrete version: the theory can be developed equally well using finite differences in place of derivatives if no suitable choice of $l(x)$ can be found; i.e. one which satisfies (L2). In this case (L2) is replaced by the corresponding $r^{\text {th }}$-difference:

$$
\Delta^{r} l_{n+m}=O\left(\left|\Delta^{r} l_{n}\right|\right) \quad \text { for } m=O(\sqrt{n} \log n) .
$$

where $\Delta a_{n}=a_{n}-a_{n-1}$ and $\Delta^{r}=\Delta^{r-1}(\Delta)$. Then we have the 'Taylor development' for such $m$

$$
l_{n+m}=l_{n}+\left(\begin{array}{c}
m \\
1
\end{array}\right) \Delta l_{n}+\left(\begin{array}{c}
m+1 \\
2
\end{array}\right) \Delta^{2} l_{n}+\ldots+\left(\begin{array}{c}
m+r-2 \\
r-1
\end{array}\right) \Delta^{r-1} l_{n}+O\left(|m|^{r}\left|\Delta^{r} l_{n}\right|\right) .
$$

4. The polynomials $Q_{k}(n)$ and $q_{k}(n)$.

From the proof of Proposition 2, we can find the polynomials $Q_{k}(n)$ via the formula

$$
Q_{k}(n)=-\operatorname{Res}_{z=1}\left\{\frac{R_{k}(P(z))}{z^{n+1}}\right\}=-\operatorname{Res}_{z=1}\left\{\frac{r_{k}(P(z))}{z^{n+1}(1-P(z))^{k+1}}\right\}
$$

by expanding $r_{k}(P(z)) z^{-n-1}(1-P(z))^{-k-1}$ in powers of $z-1$ and picking out the coefficient of $(z-1)^{-1}$. Relation (3.6) can then be used to determine the polynomials $q_{k}(n)$. It is however a laborious way of finding $q_{k}(n)$ (even for small $k$ ) since it involves determining first $Q_{0}(n), \ldots, Q_{k}(n)$. Moreover, the degree of $q_{k}(n)$ is only $[k / 2]$ while the $Q_{r}(n)$ have degree $r$, so this method will mean a lot of unnecessary work. Even determining $Q_{k}(n)$ from (4.1) is not straightforward as it involves expanding $r_{k}(P(1+t))$ in terms of powers of $t$, for which we first must find $r_{k}(\cdot)$. A more efficient method is called for.

\section{Proposition 6}

We have

$$
\begin{aligned}
Q_{k}(n) & =\left[\frac{1}{t}\right] \frac{(-1)^{k} k !(\log P(1+t))^{-k-1}}{(1+t)^{n+1}} \\
q_{k}(n) & =\left[\frac{1}{t}\right] \frac{(-1)^{k} P(1+t)^{\sigma n}}{(\log P(1+t))^{k+1}(1+t)^{n+1}} .
\end{aligned}
$$


Proof. From (4.1), we have

$$
Q_{k}(n)=-\operatorname{Res}_{z=1}\left\{\frac{R_{k}(P(z))}{z^{n+1}}\right\}=\left[\frac{1}{t}\right]-\frac{R_{k}(P(1+t))}{(1+t)^{n+1}}
$$

where $R_{k}(x)=\sum_{r=0}^{\infty} r^{k} x^{r}$. Now, it is known that ${ }^{4} R_{k}(x)=k !(-\log x)^{-k-1}+O(1)$ as $x \rightarrow 1-$. Note that as $x \rightarrow 1-, P(x) \rightarrow 1$ from below as well, so

$$
R_{k}(P(x))=k !(-1)^{k+1}(\log P(x))^{-k-1}+O(1) \quad \text { as } x \rightarrow 1-.
$$

Let $x=1+t$. Therefore

$$
-\frac{R_{k}(P(1+t))}{(1+t)^{n+1}}=\frac{(-1)^{k} k !(\log P(1+t))^{-k-1}}{(1+t)^{n+1}}+O(1) \quad \text { as } t \rightarrow 0-.
$$

The first term on the RHS has an asymptotic expansion as $t \rightarrow 0-$ and hence it must coincide for negative powers of $t$ with the asymptotic expansion of the LHS. In particular, the coefficients of $1 / t$ coincide. This proves (i).

For (ii), use part (i) and substitute into relation (3.6). Thus

$$
\begin{aligned}
q_{k}(n) & =\left[\begin{array}{l}
\frac{1}{t} \\
k
\end{array} \quad \frac{1}{k !} \sum_{r=0}^{k}\left(\begin{array}{l}
k \\
r
\end{array}\right)(-\sigma n)^{k-r}(-1)^{r} r ! \frac{(\log P(1+t))^{-r-1}}{(1+t)^{n+1}}\right. \\
& =\left[\frac{1}{t}\right] \quad \frac{(-1)^{k}(\log P(1+t))^{-k-1}}{(1+t)^{n+1}} \sum_{r=0}^{k} \frac{(\sigma n \log P(1+t))^{r}}{r !} \\
& =\left[\frac{1}{t}\right] \quad \frac{(-1)^{k}}{(\log P(1+t))^{k+1}(1+t)^{n+1}} \cdot\left\{e^{\sigma n \log P(1+t)}+O\left((\log P(1+t))^{k+1}\right)\right\} \\
& =\left[\frac{1}{t}\right] \quad \frac{(-1)^{k} P(1+t)^{\sigma n}}{(\log P(1+t))^{k+1}(1+t)^{n+1}}+O(1) \quad \text { as } t \rightarrow 0-.
\end{aligned}
$$

Again, the $O(1)$ term has no effect on the coefficient of $1 / t$ and (ii) follows.

Proposition 6 allows us to calculate the $q_{k}(n)$ quite efficiently. It is convenient to let $h(t)=$ $\frac{\sigma}{t} \log P(1+t)$, so that (asymptotically, for $t$ small and negative)

$$
h(t) \approx \frac{\sigma}{t} \log \left(1+\frac{t}{\sigma}+\frac{P^{\prime \prime}(1) t^{2}}{2}+\ldots\right)=1+\alpha_{1} t+\alpha_{2} t^{2}+\ldots=\sum_{n=0}^{\infty} \alpha_{n} t^{n}
$$

say. Thus $\alpha_{0}=1$ and $\alpha_{1}=\sigma \tau-\frac{1}{2}$, so $\alpha_{1}+\frac{1}{2}=\sigma \tau>0$. Proposition 6(ii) then tells us that

$$
q_{k}(n)=\left[t^{k}\right] \quad \frac{(-1)^{k} \sigma^{k+1}}{(1+t) h(t)^{k+1}} \exp \{n(t h(t)-\log (1+t))\} .
$$

Note that

$$
\exp \{n(t h(t)-\log (1+t))\}=1+n\left(\alpha_{1}+1 / 2\right) t^{2}+n\left(\alpha_{2}-1 / 3\right) t^{3}+\ldots
$$

$$
{ }^{4} R_{k}\left(e^{-y}\right)=(-1)^{k} \frac{d^{k}}{d y^{k}} \frac{1}{1-e^{-y}}=(-1)^{k} \frac{d^{k}}{d y^{k}}\left(\frac{1}{y}+a_{1}+a_{2} y+\ldots\right)=\frac{k !}{y^{k+1}}+O(1) \text { as } y \rightarrow 0 .
$$


while

$h(t)^{-k-1}=\left(1+\alpha_{1} t+\alpha_{2} t^{2}+\ldots\right)^{-k-1}=1-(k+1) \alpha_{1} t+\left(\left(\begin{array}{c}k+2 \\ 2\end{array}\right) \alpha_{1}^{2}-(k+1) \alpha_{2}\right) t^{2}-\ldots$

This gives $q_{k}(n)$ for $k=0,1,2,3$ as follows:

$$
\begin{aligned}
& q_{0}(n)=\sigma \\
& q_{1}(n)=\sigma^{2}\left(1+2 \alpha_{1}\right) \\
& q_{2}(n)=\sigma^{3}\left(\frac{1}{2}+\alpha_{1}\right) n+\sigma^{3}\left(1+3 \alpha_{1}-3 \alpha_{2}+6 \alpha_{1}^{2}\right) \\
& q_{3}(n)=\sigma^{4}\left(\frac{5}{6}+3 \alpha_{1}+4 \alpha_{1}^{2}-\alpha_{2}\right) n+\sigma^{4}\left(1+4 \alpha_{1}-4 \alpha_{2}+4 \alpha_{3}+10 \alpha_{1}^{2}-20 \alpha_{1} \alpha_{2}+20 \alpha_{1}^{3}\right) .
\end{aligned}
$$

We can also find the leading term in the polynomial $q_{k}(n)$. If $k$ is even, then the term $n\left(\alpha_{1}+1 / 2\right) t^{2}$ in the exponent $n(t h(t)-\log (1+t))$ will give rise to the leading term. Thus

$$
q_{2 k}(n)=\frac{\sigma^{2 k+1}}{k !}\left(\alpha_{1}+1 / 2\right)^{k} n^{k}+O\left(n^{k-1}\right),
$$

showing that $q_{2 k}(n)$ has degree $k$ exactly. If $k$ is odd, the leading term is somewhat more complex:

$q_{2 k-1}(n)=\frac{\sigma^{2 k}}{(k-1) !}\left(\alpha_{1}+1 / 2\right)^{k-2}\left(\left(2 k \alpha_{1}+1\right)\left(\alpha_{1}+1 / 2\right)-(k-1)\left(\alpha_{2}-1 / 3\right)\right) n^{k-1}+O\left(n^{k-2}\right)$.

\section{Applications}

(i) First we illustrate Theorem 1 by taking $l_{n}=n^{\lambda}(n \in \mathbb{N}, \lambda \in \mathbb{R}), l_{0}=1$ when $\lambda=0$, $l_{0}=0$ otherwise. Thus we have

$$
f_{n}=\sum_{m=0}^{\infty} m^{\lambda} p_{n}^{* m} .
$$

(The series starts from 1 if $\lambda \neq 0$ ). The cases $\lambda=0$ and $\lambda=-1$ correspond to renewal and harmonic renewal theory respectively. Here we may interpolate $l_{n}$ by $l(x)=x^{\lambda}$ for $x>0$. Then $l^{(k)}(x)=k !\left(\begin{array}{l}\lambda \\ k\end{array}\right) x^{\lambda-k}$. This satisfies (L2) (for $t=o(x)$ ). Hence by Theorem 1 , if $p_{n}$ is non-negative, aperiodic and $O\left(e^{-\eta \sqrt{n}}\right)$ for some $\eta>0$, we have the asymptotic expansion

$$
f_{n} \approx(\sigma n)^{\lambda} \sum_{k=0}^{\infty} k !\left(\begin{array}{l}
\lambda \\
k
\end{array}\right) \frac{q_{k}(n)}{\sigma^{k} n^{k}} \approx n^{\lambda} \sum_{k=0}^{\infty} \frac{a_{k}}{n^{k}}
$$

for some coefficients $a_{k}$, with $a_{0}=\sigma^{\lambda+1}$. Using the formulae for $q_{k}(n)$ and writing $\sigma \tau$ for $\alpha_{1}+\frac{1}{2}$, we find

$$
f_{n}=\sigma^{\lambda+1} n^{\lambda}\left\{1+\frac{\lambda(\lambda+1) \sigma \tau}{n}+\frac{\lambda\left(\lambda^{2}-1\right)}{2 n^{2}}\left((\lambda+2) \sigma^{2} \tau^{2}-2 \sigma \tau-2 \alpha_{2}+\frac{7}{6}\right)+O\left(\frac{1}{n^{3}}\right)\right\} .
$$

Of course, the case where $\lambda$ is a non-negative integer (when $\left(\begin{array}{l}\lambda \\ k\end{array}\right)=0$ for $k \geq \lambda$ ) is already dealt with in Proposition 2 under the weaker condition that $p_{n}=O\left(n^{-A}\right)$ for all $A$. The leading term agrees with the known results in [4] and [11], though these were proven subject 
to much weaker conditions. The discrepancy in bounds on $p_{n}$ arises from the fact that in Theorem 1, we allow $l_{n}$ to be as large/small as $e^{ \pm n^{1 / 2-\varepsilon}}$, for which we need the stronger bound on $p_{n}$.

\section{(ii) Bernoulli transforms}

For a given sequence of real numbers $\left\{l_{k}\right\}$, the Bernoulli transform of $l_{k}$ with parameter $p \in(0,1)$ is defined as

$$
S_{n}=\sum_{k=0}^{n}\left(\begin{array}{l}
n \\
k
\end{array}\right) l_{k} p^{k} q^{n-k} \quad \text { where } q=1-p .
$$

The problem here is to find the asymptotic behaviour of $S_{n}$ as $n \rightarrow \infty$, given the sequence $l_{k}$. This problem was considered by Flajolet in [5] who estimated $S_{n}$ asymptotically for a number of special cases with $l_{k}$ of at most polynomial growth using singularity analysis. In this section, we use Theorem 1 to obtain an asymptotic expansion for $S_{n}$. Our method applies in general and the $l_{k}$ are not restricted in size to polynomial growth. Furthermore, we do not require an analytic continuation of the generating function of $l_{k}$ beyond the unit disc.

Flajolet pointed out that if $L(z)$ and $S(z)$ are the generating functions of $l_{n}$ and $S_{n}$ respectively, then

$$
S(z)=\frac{1}{1-q z} L\left(\frac{p z}{1-q z}\right) .
$$

The RHS is of the form $\frac{1}{p z} L_{1}(P(z))$ where $L_{1}(z)=z L(z)$ and $P(z)=\frac{p z}{1-q z}$. Now, $P(z)=$ $\sum_{n=1}^{\infty} p q^{n-1} z^{n}$ which is analytic for $|z|<1 / q$, is not of the form $k\left(z^{r}\right)$ for any $r \geq 2$ and $P(1)=1$. Hence, if we assume sufficiently smooth behaviour for $l_{n}$, we can apply our results. Here $P^{\prime}(1)=1 / p$ and so $\sigma=p$. We note first that on the single assumption that $l_{n+[\lambda \sqrt{n}]} \sim l_{n}$ for all $\lambda$, we have

$$
S_{n} \sim l_{[p n]} \quad \text { as } n \rightarrow \infty .
$$

To obtain more detailed asymptotic behaviour assume further that $l_{n}$ satisfies (L2) (which implies $l_{n-1}$ satisfies (L2) also). Thus, by Theorem 1, we have

$$
\left[z^{n}\right] L_{1}(P(z))=\sum_{k=0}^{r-1} l^{(k)}(p n-1) q_{k}(n)+O\left(\left|l^{(r)}(p n-1)\right| n^{r / 2}\right)+O\left(\frac{l(p n-1)}{n^{A}}\right) \quad \text { for all } A \text {. }
$$

In this case

$$
h(t)=\frac{\sigma}{t} \log P(1+t)=\frac{p}{t} \log \left(\frac{1+t}{1-\frac{q}{p} t}\right)=\sum_{k=0}^{\infty} \frac{1}{k+1}\left((-1)^{k} p+q^{k+1} p^{-k}\right) t^{k},
$$

so that $\alpha_{k}=\frac{1}{k+1}\left((-1)^{k} p+q^{k+1} p^{-k}\right)$. Substituting into the formulas for $q_{k}(n)$ gives

$$
\begin{aligned}
& q_{0}(n)=p \\
& q_{1}(n)=p q \\
& q_{2}(n)=\frac{1}{2} p^{2} q n+\frac{1}{2} p q(q-p) \\
& q_{3}(n)=\frac{1}{6} p^{2} q(4 q-p) n+\frac{1}{6} p q\left(q^{2}-4 p q+p^{2}\right) \\
& q_{4}(n)=\frac{1}{8} p^{3} q^{2} n^{2}+O(n) .
\end{aligned}
$$


Hence, since $S_{n}=\frac{1}{p}\left[z^{n+1}\right] L_{1}(P(z))$, this gives the asymptotic expansion

$$
\begin{aligned}
S_{n} & =\frac{1}{p} \sum_{k=0}^{r-1} l^{(k)}(p n-q) q_{k}(n+1)+O\left(\left|l^{(r)}(p n-q)\right| n^{r / 2}\right)+O\left(\frac{l(p n)}{n^{A}}\right) \quad(\text { for all } A) \\
& \approx l(p n-q)+q l^{\prime}(p n-q)+\frac{q}{2}(p n+q) l^{\prime \prime}(p n-q)+\frac{q}{6}\left(p(4 q-p) n+q^{2}\right) l^{\prime \prime \prime}(p n-q)+\ldots
\end{aligned}
$$

(iii) Negative Binomial Sums

In a similar fashion, we can deal with 'negative' Binomial sums which were studied in [9] in connection with entropy calculations. For a given sequence $\left\{l_{k}\right\}_{k \geq 0}$ and $p \in(0,1)$, let

$$
f_{n}=\sum_{k \geq n}\left(\begin{array}{l}
k \\
n
\end{array}\right) p^{n} q^{k-n} l_{k} \quad(n \geq 0)
$$

where $q=1-p$. Again, we are interested in the behaviour of $f_{n}$ as $n \rightarrow \infty$. Here we have (formally)

$$
\begin{aligned}
F(z) & \stackrel{\text { def }}{=} \sum_{n=0}^{\infty} f_{n} z^{n}=\sum_{n=0}^{\infty} \sum_{k=n}^{\infty}\left(\begin{array}{l}
k \\
n
\end{array}\right) p^{n} q^{k-n} l_{k} z^{n} \\
& =\sum_{k=0}^{\infty} l_{k} \sum_{n=0}^{k}\left(\begin{array}{l}
k \\
n
\end{array}\right) p^{n} q^{k-n} z^{n}=\sum_{k=0}^{\infty} l_{k}(p z+q)^{k}=L(P(z))
\end{aligned}
$$

where $L(z)=\sum_{n=0}^{\infty} l_{n} z^{n}$ and $P(z)=q+p z$. An asymptotic expansion for $f_{n}$ can therefore readily be found under the appropriate conditions by applying Theorem 1 . The function $P(z)$ satisfies the necessary conditions and $\sigma=1 / p$. In this case, $\frac{\sigma}{t} \log P(1+t)=\frac{1}{p t} \log (1+p t)$ so that $\alpha_{k}=\frac{(-1)^{k} p^{k}}{k+1}$ from which the $q_{k}(n)$ are found to be

$$
\begin{gathered}
q_{0}(n)=\frac{1}{p}, \quad q_{1}(n)=\frac{q}{p^{2}}, \quad q_{2}(n)=\frac{q}{2 p^{3}}(n+2-p), \\
q_{3}(n)=\frac{q}{6 p^{4}}\left((5-4 p) n+p^{2}-6 p+6\right), \quad q_{4}(n)=\frac{q^{2}}{8 p^{5}} n^{2}+O(n), \ldots
\end{gathered}
$$

Thus, assuming $l_{n}$ satisfies (L1) and (L2), we have the asymptotic expansion

$f_{n} \approx \frac{1}{p} l\left(\frac{n}{p}\right)+\frac{q}{p^{2}} l^{\prime}\left(\frac{n}{p}\right)+\frac{q}{2 p^{3}}(n+2-p) l^{\prime \prime}\left(\frac{n}{p}\right)+\frac{q}{6 p^{4}}\left((5-4 p) n+p^{2}-6 p+6\right) l^{\prime \prime \prime}\left(\frac{n}{p}\right)+\ldots$

(iv) In [1], Baltrūnas and Šiaulys considered the case where the $l_{n}$ are of the form $e^{-H_{n}}$ and where the 'hazard' sequence, $h_{n}:=H_{n}-H_{n-1}$, is decreasing and satisfies $n h_{n} \leq\left(\frac{1}{2}-\delta\right) H_{n}$ for some $\delta>0$ (for large $n$ ). They found that

$$
f_{n}=\sigma l_{[\sigma n]}\left(1+O\left(\frac{H_{n} \log n}{\sqrt{n}}\right)\right)
$$

under the assumption that $p_{n}=O\left(e^{-\lambda n}\right)$ for some $\lambda>0$. Here we show that the $\log n$ may be omitted, even under the weaker condition (P3). For we have, for $n>m$

$$
\frac{l_{m}}{l_{n}}=\exp \left\{\sum_{r=m+1}^{n} h_{r}\right\}
$$


and since $h_{r}$ decreases eventually, it easily follows that for $m, n$ sufficiently large

$$
\exp \left\{(n-m) h_{n}\right\} \leq \frac{l_{m}}{l_{n}} \leq \exp \left\{(n-m) h_{m}\right\}
$$

As $l_{n}$ was shown in [1] to satisfy (L1), it follows (on (P1) and (P3)) that (3.7) holds and hence $f_{n}=\sigma l_{[\sigma n]}+S_{n}+O\left(\frac{l_{[\sigma n]}}{n^{A^{\prime}}}\right)\left(A^{\prime}\right.$ as before $)$, where

$$
S_{n}=\sum_{|\Delta| \leq A \sqrt{n} \log n}\left(l_{m}-l_{[\sigma n]}\right) p_{n}^{* m} .
$$

However, for $\Delta=m-\sigma n=O(\sqrt{n} \log n)$, both $h_{m}$ and $h_{[\sigma n]}$ are $O\left(H_{n} / n\right)=O\left(n^{-\alpha}\right)$ for some $\alpha>\frac{1}{2}$ (see [1]), so $\Delta h_{m}$ and $\Delta h_{[\sigma n]}$ are both $o(1)$. Hence (5.1) implies

$$
l_{m}=l_{[\sigma n]}\left(1+O\left(\frac{|\Delta| H_{n}}{n}\right)\right),
$$

which in turn implies (by the estimates in the proof of Theorem 1)

$$
\left|S_{n}\right|=O\left(\frac{l_{[\sigma n]} H_{n}}{n} \sum_{|\Delta| \leq A \sqrt{n} \log n}|\Delta| p_{n}^{* m}\right)=O\left(\frac{l_{[\sigma n]} H_{n}}{\sqrt{n}}\right) .
$$

It follows that

$$
f_{n}=\sigma l_{[\sigma n]}\left(1+O\left(\frac{H_{n}}{\sqrt{n}}\right)\right)
$$

Better bounds are possible under some extra conditions. For example, if $l_{m}=l_{[\sigma n]}(1-$ $\left.\Delta h_{[\sigma n]}+O\left(\Delta^{2} h_{[\sigma n]}^{2}\right)\right)$ holds for $\Delta=O(\sqrt{n} \log n)$ then

$$
\begin{aligned}
S_{n} & =-l_{[\sigma n]} h_{[\sigma n]} \sum_{|\Delta| \leq A \sqrt{n} \log n} \Delta p_{n}^{* m}+O\left(l_{[\sigma n]} h_{[\sigma n]}^{2} \sum_{|\Delta| \leq A \sqrt{n} \log n} \Delta^{2} p_{n}^{* m}\right) \\
& =O\left(l_{[\sigma n]} h_{[\sigma n]}\right)+O\left(n l_{[\sigma n]} h_{[\sigma n]}^{2}\right)=O\left(\frac{l_{[\sigma n]} H_{n}}{n}\right),
\end{aligned}
$$

giving $f_{n}=\sigma l_{[\sigma n]}\left(1+O\left(\frac{H_{n}^{2}}{n}\right)\right)$.

Consider their specific example where $p_{n}=\frac{1}{n ! e}$ and $l_{n}=e^{-\sqrt[3]{n}}$. In this case, we can let $l(x)=e^{-\sqrt[3]{x}}$. It is easily seen that

$$
l^{(k)}(x)=(-3)^{-k} x^{-\frac{2 k}{3}} e^{-\sqrt[3]{x}} P_{k}\left(x^{-1 / 3}\right),
$$

where $P_{k}(\cdot)$ is a polynomial with $P_{k}(0)=1$. Thus (L2) is satisfied (for $t=O\left(x^{2 / 3}\right)$ ). We have $\sigma=1$ and Theorem 1 gives the asymptotic expansion

$$
f_{n} \approx \sum_{k=0}^{\infty} q_{k}(n) l^{(k)}(n) \approx e^{-\sqrt[3]{n}} \sum_{k=0}^{\infty} \frac{a_{k}}{n^{k / 3}},
$$

for some constants $a_{k}$. An easy calculation gives $a_{0}=1, a_{1}=\frac{1}{18}$ and $a_{2}=-\frac{2}{9}$.

Acknowledgement. I wish to thank the referees for some useful suggestions. 


\section{References}

[1] A. Baltrūnas and J. Šiaulys, Second order asymptotic behaviour of subordinated sequences with longtailed subordinator, Journal of Mathematical Analysis and Applications, 332 (2007) 22-31.

[2] N. H. Bingham, C. M. Goldie, and J. L. Teugels, Regular variation, Cambridge University Press, 1987.

[3] N. G. de Bruijn, Asymptotic Methods in Analysis, North-Holland Publishing Co., 1970 (3rd edition).

[4] P. Embrechts, M. Maejima, and E. Omey, A renewal theorem of Blackwell type, Ann. Prob. 12 (1984), 561-570.

[5] P. Flajolet, Singularity analysis and asymptotics of Bernoulli sums, Theoretical Computer Science 215 (1-2) (1999) 371-381.

[6] P. Flajolet and A.M. Odlyzko, Singularity analysis of generating functions, SIAM J. Discrete Math. 3 (1990) 216-240.

[7] R. Grübel, On subordinated distributions and generalised renewal measures, Ann. Prob. 15 (1987) 394-415.

[8] T. W. Hilberdink, On the Taylor coefficients of the composition of two analytic functions, Ann. Acad. Sc. Fenn. Mathematica 21 (1996) 189-204.

[9] P. Jacquet and W. Szpankowski, Entropy computations via analytic depoissonization, IEEE Trans. Inform. Theory 45 (1999) 1072-1081.

[10] C. Knessl, Integral representations and asymptotic expansions for Shannon and Renyi Entropies, Appl. Math. Lett. 11 No.2 (1998) 69-74.

[11] M. Maejima and E. Omey, A generalized Blackwell renewal theorem, Yokohama Math. Journal 32 (1984), 123-133.

[12] A. M. Odlyzko, Asymptotic enumeration methods, Handbook of Combinatorics Vol. 2, Elsevier, 1995. 